\title{
The role of galectin proteins in the induction of apoptosis in arthritic joints; routes for new therapies for autoimmune diseases and cancer
}

\begin{abstract}
Rheumatoid arthritis (RA) is a common chronic inflammatory arthropathy, leading to joint destruction and disability as a consequence of the chronic inflammatory processes. The etiology is unknown and the pathogenesis of this disorder is not well understood, yet the molecular events leading to tissue inflammation resulting with cartilage and bone destruction are now better defined. As a result there is a better chance now than ever before to develop new therapeutic modalities; an example of such an effort is reported here. We have found new variant of human galectin- 8 protein expressed in the joints of arthritic patients. This protein induces apoptosis in treated cells and has approximately $80 \%$ therapeutic effect in CIA mouse model.
\end{abstract}

Volume 2 Issue I - 2015

Itshak Golan

Daniel \& Daisy Novel Therapeutics Ltd., United Kingdom

Correspondence: Itshak Golan, Daniel \& Daisy Novel Therapeutics Ltd., 49 Southern down Avenue, Mayals, Swansea SA3 5EL, United Kingdom, Tel 44-I792-404-I 22 +44-77-91235930,Email I.Golan@swansea.ac.uk

Received: February 10, 2015 | Published: March 05, 2015

Keywords: rheumatoid arthritis, autoimmune inflammatory diseases, disease specific biomarkers, novel biological drugs

Abbreviations: RA, rheumatoid arthritis; DMARDs, diseasemodifying anti rheumatic drugs; APCs, antigen presenting cells; MHC, major histo compatibility; TCR, T cell receptor; ECM, extracellular matrix; CRDs, carbohydrate-recognition domains

\section{Introduction}

Rheumatoid Arthritis (RA) is a chronic, systemic inflammatory disease primarily involving the joints. It affects $\sim 1 \%$ of the population with a female to male ratio of $3: 1$. The disease causes a severe morbidity, suffering as well significant economic damage by lots of labor days and expensive treatment protocols. Therapy with slowacting, disease-modifying anti rheumatic drugs (DMARDs), such as low-dose methotrexate, which is generally accepted as a standard treatment, may achieve amelioration of symptoms, but usually does not eradicate long-term joint destruction. Similar considerations are true for all other pharmacological options of this drug family. As a result, therapeutic limitations on the one hand, and the increasing knowledge of inflammatory processes and mediators as therapeutic targets, on the other hand, have encourage the development of novel RA therapeutic agents, such as monoclonal antibodies, cytokinereceptor / human-immunoglobulin constructs or recombinant human proteins. Clinical trials of TNF - $\alpha$ blocking agents, alone or in combination with methotrexate, have convincingly shown the feasibility and efficacy of these novel approaches to the therapy of RA. However, even these biologic modalities show a response rate of $\sim 60-70 \%$. Their safety profile carries an increased risk of severe infections and possibly malignancy, and their direct costs are high. These limitations encourage the development of new therapeutic modalities.

We are reporting here our recent findings on new pro apoptotic agents, galectin- 8 proteins, which can provide novel diagnostic, prognostic and therapeutic strategies for RA patients and other joint / chronic inflammatory diseases. Galectins comprise a family of proteins that are widely expressed in mammals. All galectins share the ability to bind $\alpha$-galactosides through an evolutionarily conserved sequence motif in the carbohydrate-binding site. ${ }^{1}$ They have a cytoplasmic or nuclear localization and can be externalized - despite the fact that they lack a single peptide - as soluble proteins by non-classical secretory mechanisms. ${ }^{2,3}$ In mammals, 15 galectins have been characterized. ${ }^{3,4}$ Two groups of galectins are distinguished according to the number of lectin domains. Most of them, including galectin-1, 2, 3, 5, 7 and 10 , have a single lectin domain. Galectin-4, 6, 8 and 9 belong to the second group and are characterized by two tandem arranged lectin domains linked by a hinged peptide. ${ }^{4-7}$

The exact role(s) of galectins is not yet clearly established, but they are suspected to modulate several cellular functions such as physiological $^{8}$ and malignant cell adhesion, cell proliferation, ${ }^{9}$ apoptosis, ${ }^{10}$ metastasis seeding ${ }^{11}$ and immune function. ${ }^{12,13}$ They have also been described as nuclear proteins ${ }^{14}$ possibly involved in pre-messenger RNA splicing. ${ }^{15,16}$ Expression analysis has revealed a broad distribution in healthy tissues for galectin-1, 3, 4, 8 and 9 , while galectin-2, 5, 6 or 7 are restricted mainly to one specific tissue (such as lung, prostate etc.). ${ }^{6,17}$ Differential expression of these proteins has been well documented in the literature in healthy tissues or in tumor cells. ${ }^{9}, 11,18$ In previous communication ${ }^{19}$ we have shown that galectin- 8 induces apoptosis in synovial fluid inflammatory cells of rheumatoid arthritis (RA) patients (which are involved in the destruction of bone and cartilage) and we further found that soluble CD44 (which can be detected in the synovium) naturalize this program cell death.

Therefore, the balance between galectin- 8 and soluble CD44 may dictate the intensity of the inflammatory cascade. The aim of the study described in this communication was to explore in more detail the ability of RA-related galectin- 8 variants to induce apoptotic signal in cells derived from synovial membrane and fluid of arthritic patients, to compare this effect with the ability of the galectin- 8 to induce apoptosis in peripheral blood leukocytes and finally to examine the therapeutic potential of this mammal's lectin in a mouse model of collagen induced arthritis.

\section{Discussion}

Selective therapeutic targeting of pathological cells, such as inflammatory or cancer cells, has, as in previous decades, remained a major challenge for modern medicine. Even minor differences in cell surface molecules or signal transduction pathways between pathological cells and their normal counterparts could be used as a handle for selective destruction of the former. Unfortunately, the 
existence of such discrete target entities in pathological cells is not as obvious a phenomenon as predicted by many investigators. Even the targeting of idiotypes (on B cell lymphomas) or tumor-associated antigens (including oncogene products), which have been considered the most promising candidates for specific therapy, have not advanced beyond "experimental trials," although the concept has been discussed for more than two decades.

Discrimination between "harmful" inflammatory cells, involved in self-destruction, and inflammatory cells engaged in the eradication of microorganisms is no less complicated, because there is no indication that the two cell types differ chemically or biologically. Again, the discriminatory elements might be found in the T cell receptor (TCR) of those cells initiating the inflammatory cascade or in the corresponding major histo compatibility (MHC) sequences of antigen presenting cells (APCs), which bind the relevant peptide. This approach, which has been successfully applied over the past few years in some animal models of autoimmunity, has not been tested clinically, except in several experimental trials. Unfortunately, targeting of TCR or MHC molecules would be effective only at the initial phase of autoimmune diseases, when the symptoms are still undetectable. As a consequence, in many cases the approach may be impractical.

A different tactic is to target proinflammatory molecules that are involved in the later, already detectable, exacerbated stage of the process, when there is a non-specific influx of leukocytes, attracted by chemokines and activated by cytokines. Indeed, it has been shown that autoimmune diseases, both in animals and humans, can be controlled by targeting inflammation-supportive molecules, such as selectins, integrins, IL-12, TNFa and NFkB, using specific antibodies or anti-sense oligonucleotides. However, the same molecules are also involved in essential physiological functions and, therefore, we can never know when and how the price of such "nonspecific" therapy will be exacted. This long-lasting dilemma raises the question: can we define an essential proinflammatory molecule, involved in a relatively late phase of the process that displays sufficient chemical diversity to allow restricted targeting? As we shall see here, the galectin- 8 proteins may meet this requirement.

Whereas the function of selectins and integrins in supporting cancer and inflammatory cell migration and docking in target organs has been well documented, the role of galectins and especially galectin- 8 has only recently attracted attention. Integrins are a family of $\mathrm{a}$ and $\mathrm{b}$ subunits where each $\mathrm{b}$ subunit complexes with a distinct set of a subunits to create heterodimers with characteristic binding specificities. The topographies of $\mathrm{a}$ and $\mathrm{b}$ subunits are similar. The majority of each is extracellular, and amino-terminal regions, mainly of the subunits, comprise the ligand-binding pocket. Ligand binding, cell adhesion and other properties of integrins require divalent cations. Most integrins bind Ligands that are components of extracellular matrix (ECM) (e.g. fibronectin, collagen and vitronectin), and both ligand occupancy and integrin-mediated responses.

One of the major functions of integrins is adhesive cell-cell and cellmatrix interactions. Cell adhesion also depends upon carbohydrateprotein interactions, mediated by lectins of different families. Selectins, a family of cell surface glycoprotein's, mediate lymphocyte homing to peripheral lymph nodes and are involved in both neutrophil and lymphocyte adhesion to activated endothelial cells. Similarly, galectins, animal lectins, which specifically bind b-galactoside residues, were implicated as modulators of cell adhesion. While lacking a signal peptide and found mainly in the cytosol, galectins are externalized by an atypical secretory mechanism to mediate cell growth, cell transformation, embryogenesis and apoptosis. In accordance with their proposed functions, galectins enhance or inhibit cell-matrix interactions. Though little is known about the exact role of galectins in inhibiting cell adhesion, current models suggest that binding of galectins to matrix proteins elicits an inhibitory effect due to steric hindrance of cell-matrix interactions.

Galectin 8, a member of the galectin family, is a $67 \mathrm{kDa}$ secreted protein, made of two homologous (38\% identity) carbohydraterecognition domains (CRDs) linked by a short (approx. 26 amino acids) linking peptide. It is already showed that galectin- 8 is an integrin binding-protein that interacts with several, but not all, members of integrin family. A conformational change induced upon binding of galectin- 8 to integrins presumably accounts for the inhibitory effects of galectin- 8 on cell adhesion and its ability to induce apoptosis. These events do not involve a steric hindrance of cell-matrix interaction, but rather result from anti-adhesive effects of galectin- 8 . Thus, galectin- 8 represents a novel class of cell adhesion modulators that bind sugar moieties of integrins to regulate integrin interactions with the extracellular matrix and the signal generated thereof. In conclusion, galectin-8 acts as an integrin binding-protein that exerts downmodulatory effects on integrin receptor functions. The anti-adhesive effects induced by galectin- 8 are specific and little evidence, which will not describe here, support this evidence. Thus, the interaction of galectin-8 with integrins seems to be of physiological relevance.

Inhibition of martrix-integrins interactions has been implicated in the induction of apoptosis in cells denied of anchorage. We could demonstrate that galectin- 8 is effective inducer of an apoptotic process in 1299 cells. Apoptosis, like binding to integrins, was prevented upon inclusion of TDG, suggesting that both events were at least partially mediated by interactions of galectin- 8 with cell surface glycoconjugates. However, apoptosis induced by galectin- 8 was not secondary to its anti-adhesive signals, since it took place when galectin- 8 was applied to adherent cells that did not detach during the course of the experiment. Moreover, and in the contrast to inhibition of cell adhesion, which occurred in the presence of serum, apoptosis induced by galectin- 8 occurred only in a serum-free medium. These findings indicate that galectin- 8 might induce its anti-adhesive and apoptotic effects by two independent mechanisms; furthermore, serum constituents might act as survival factors to rescue the cells from apoptotic signals transmitted by galectin- 8 .

Once having established this finding, we should be able to move one step further in an attempt to target, using galectin- 8 or variant isoform of this protein, the inflammatory cells involved in rheumatoid arthritis and other arthritic inflammatory conditions. By local blocking the activity of these cells, by inducing apoptotic pathways, inflammatory arthritic diseases may be inhibited without affecting normal physiological functions (galectin- 8 is nature molecule in the body).

\section{Conclusion}

We can say that our recent findings indicates a new pro-apoptotic direction, using the novel galectin isoform derived from synovial fluids of RA patients, which provide a novel diagnostic, prognostic and therapeutic approach for RA and other joint / chronic inflammatory diseases.

\section{Acknowledgments}

None.

\section{Conflicts of interest}

Author declares there are no conflicts of interest. 


\section{Funding}

None.

\section{References}

1. Barondes S, Castronovo V, Cooper D, et al. Galectins: a family of animal b-galactoside-binding lectins. Cell. 1994;76(4):597-598.

2. Cooper DN, Barondes SH. Evidence for export of a muscle lectin from cytosol to extracellular matrix and for a novel secretory mechanism. $J$ Cell Biol. 1990;110(5):1681-1691.

3. Barondes SH, Cooper DNW, Gitt MA, et al. Structure and function of a large family of animal lectins. J Biol Chem . 1994;269(33):20807-20810.

4. Wada J, Kanwar YS. Identification and characterization of galectin-9, a novel beta-galactoside-binding mammalian lectin. J Biol Chem. 1997;272(9): 6078-6086.

5. Drickamer K, Taylor ME. Biology of animal lectins. Annu Rev Cell Biol .1993;9:237-264

6. Gitt MA, Colnot C, Poirier F, et al. Galectin-4 and galectin-6 are two closely related lectins expressed in mouse gastrointestinal tract. $J$ Biol Chem. 1998;273(5):2954-2960.

7. Hadari Y, Paz K, Dekel K, et al. Galectin-8, a new rat lectin, related to galectin-4. J Biol Chem. 1995;270(7):3447-3453.

8. Mc Ever RP, Moore KL, Cummings RD. Leucocyte trafficking mediated by selectin-carbohydrate interactions. $J$ Biol Chem. 1995;270(19):11025-11028.

9. Schoeppner HL, Raz A, Ho SB, et al. Expression of an endogenous galactose-binding lectin correlates with neoplastic progression in the colon. Cancer. 1995;75(12):2818-2826.
10. Yang RY, Hsu DK, Liu FT. Expression of galectin-3 modulates T-cell growth and apoptosis. Proc Natl Acad Sci USA 1996;93(13):6737-6742.

11. Bresalier RS, Byrd JC, Wang L, et al. Colon cancer mucin: a new ligand for the beta-galactoside-binding protein galectin-3. Cancer Res . 1996;56(19):4354-4357.

12. Cherayil BJ, Chaitovitz S, Wong C, et al. Molecular cloning of human macrophage lectin specific for galactose. Proc Natl Acad Sci U S A. 1990;87(18):7324-7328.

13. Liu FT. S-type mammalian lectins in allergic anflammation. Immunol Today. 1993;14(10):486-490.

14. Jia S, Wang JL. Carbohydrate binding protein 35: complementary DNA sequence reveals homology with proteins of the heterogeneous nuclear RNP. J Biol Chem. 1998;263(13):6009-6011.

15. Dagher SF, Wang JL, Patterson RJ. Identification of galectin-3 as a factor in pre-mRNA splicing. Proc Natl Acad Sci USA. 1995;92(4):1213-1217.

16. Vyakarnam A, Dagher S, Wang J, et al. Evidence for a role for galectin-1 in pre-mRNA splicing. Mol Cell Biol. 1997;17(8):4730-4737.

17. Leonidas DD, Vatzaki EH, Worum H, et al. Structural basis for the recognition of carbohydrates by human galectin-7. Biochemistry. 1998;37(40):13930-13940.

18. Su Z, Lin J, Shen R, et al. Surface epitope masking and expression cloning identifies the human prostate carcinoma tumor antigen PCTA1, a member of the galectin gene family. Proc Natl Acad Sci U S A. 1996;93(14):7252-7257.

19. Eshkar Sebban L, Ronen D, Levartovsky D, et al. Galectin-8, a novel ligand of CD44, is involved in joint inflammation of rheumatoid arthritis. J Immunol. 2007;179:1225-1235. 